Nicola Latronico Martin Smith

\section{Introducing simplified electrophysiological test of peripheral nerves and muscles in the ICU: choosing wisely}

Received: 4 February 2014

Accepted: 29 March 2014

Published online: 8 April 2014

(C) Springer-Verlag Berlin Heidelberg and ESICM 2014

\section{N. Latronico $(\square)$}

Department of Anesthesia and Critical Care Medicine, University of Brescia, Brescia, Italy

e-mail: latronic@med.unibs.it

\section{Smith}

Department of Neurocritical Care, University College London Hospitals, London, UK

\section{Smith}

National Institute for Health Research University College London Hospitals Biomedical Research Centre, London, UK

Critical illness polyneuropathy (CIP) and myopathy (CIM) are neuromuscular disorders that develop after admission to an intensive care unit (ICU) and which have substantial adverse effects on outcome. CIP and CIM are associated with delayed weaning from mechanical ventilation, increased ICU and hospital length of stay and associated healthcare costs, increased mortality, and protracted disability after discharge from the ICU. They therefore have enormous implications for individual patients as well as for healthcare economies.

The reported incidence of CIP and CIM is greatly influenced by the method and timing of their diagnosis, as well as the patient population [1]. In adult ICU patients with severe sepsis, septic shock, or prolonged mechanical ventilation, the incidence CIP or CIM, alone or in combination, has been variably reported between $50 \%$ [2] and $100 \%$ [3]. The definitive diagnosis of CIP and CIM relies on clinical and electrophysiological evidence, i.e., demonstration of muscle weakness utilizing the Medical Research Council (MRC) scale to assess strength in functional limb muscle groups, and nerve conduction studies (NCS) and electromyography (EMG) of individual peripheral nerves and muscles. Manual testing of muscle strength requires an awake and cooperative patient, is prevented by sedation, delirium, and consciousness disturbance, and therefore is rarely possible in the early stages of ICU treatment [4]. Components of EMG assessment can also be hampered by these confounders.

If clinical testing is impossible and the characterization of neuromuscular weakness relies only on electrophysiological studies (EPS) the diagnosis of CIP and CIM is labeled as 'probable' [5], whereas, if only clinical evaluation is possible, a diagnosis of ICU-acquired muscle weakness (ICUAW) is made [6]. However, there are limitations to both these descriptors. ICUAW does not delineate the ongoing pathological process, be it CIP, CIM, both, or neither, and offers little prognostic information. This is important because CIM has a better prognosis than CIP at both short-term [7] and long-term follow-up [8]. An article in this issue of Intensive Care Medicine by Moss and colleagues [9] highlights the importance of the accurate diagnosis of neuromuscular weakness. In this study, patients with abnormal EPS had significantly increased mortality $(50 \%)$ compared to those with normal EPS findings (13\%). A proportion of patients with normal EPS met the criteria for deconditioning but their mortality was also lower $(14 \%)$ than those with abnormal EPS. Importantly, the deconditioned patients were clinically indistinguishable from those with altered electrophysiology. Thus, EPS, along with other patient characteristics such as increasing age, greater burden of comorbid illness, and prolonged ICU length of stay, may serve to identify different phenotypes of patients with ICUAW, and thereby improve prognostication as well as guide individualized treatments [10]. For 


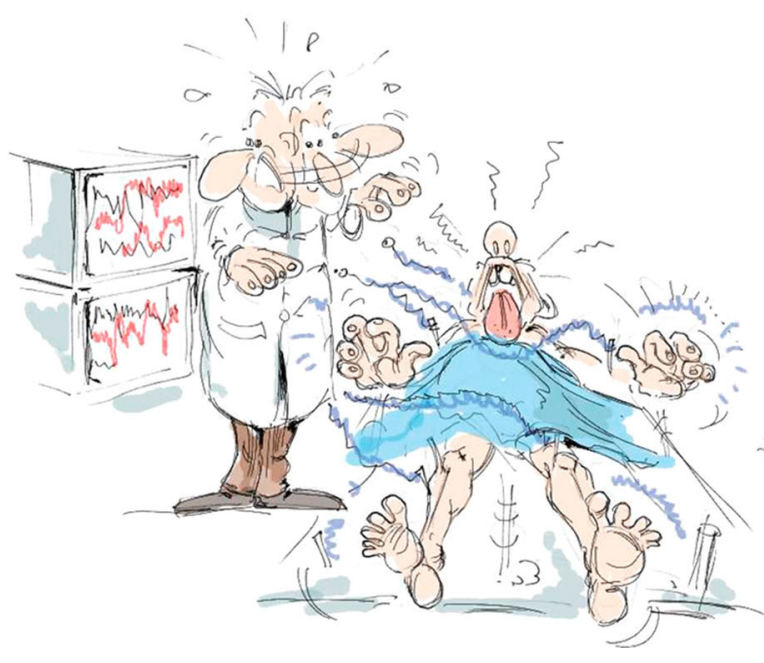

Fig. 1 Electrophysiological test of peripheral nerves and muscles

example, although the efficacy of electrical muscle stimulation is still debated $[5,11]$, it is more likely to be effective in younger patients with ICUAW and normal EPS than in the elderly with associated chronic conditions and electrically unexcitable muscle.

The clinical relevance of EPS abnormalities in the absence of clinically demonstrable muscle weakness remains unsettled. Electrophysiological abnormalities may precede muscle weakness [12], but may also resolve in the absence of demonstrable muscle weakness, possibly as other organ system dysfunction and failure, such as acute kidney injury or acute lung injury, resolve. It is uncertain whether electrophysiological abnormalities in peripheral nerves and muscles invariably precede the onset of clinically evident muscle weakness, or whether treatment-induced resolution of other organ system dysfunction or failure associated with improvements in EPS reliably leads to improved neuromuscular outcomes for the patient. Importantly, the only effective treatment for reducing the incidence of EPS-proven CIP, intensive insulin treatment to maintain normoglycemia, is associated with increased occurrence of hypoglycemia and death, and is no longer recommended [5, 13].

A comprehensive electrophysiological examination of peripheral nerves and muscles on the ICU is notoriously complex, can take up to $90 \mathrm{~min}$ to perform, and is uncomfortable for awake patients (Fig. 1). Simplified assessments of electrophysiological function that are more widely applicable in the critical care setting are therefore required. Moss and colleagues demonstrate that a simple EPS, a combination of unilateral peroneal (motor) and sural (sensory) NCS, has a $100 \%$ sensitivity and high specificity for the diagnosis of CIP/CIM. This suggests that patients with normal NCS are very unlikely to have CIP/CIM and that further testing with EMG is unlikely to alter that conclusion. On the other hand, if these NCS are abnormal, the diagnosis of CIP/CIM should be explored further. The findings of Moss et al. confirm those of

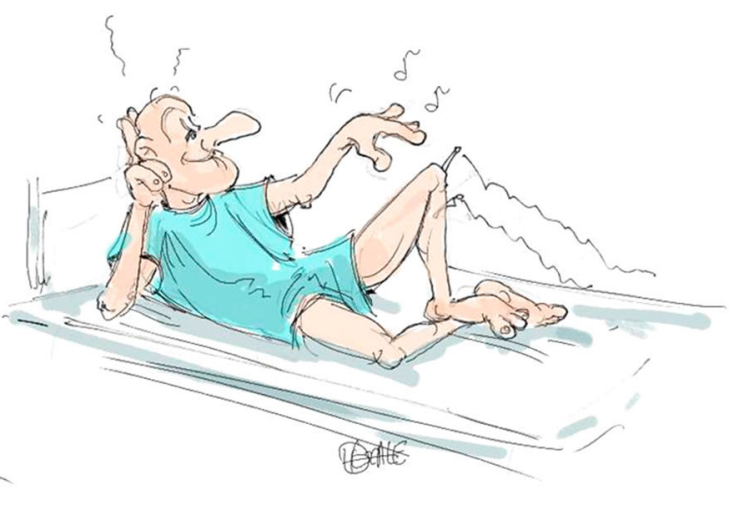

another study that also showed that the peroneal motor nerve has $100 \%$ sensitivity and moderate specificity for the diagnosis CIP or CIM [14]. On a practical note, the amplitude of the sensory nerve action potential, measured in microvolts, is 1,000 times lower than the amplitude of the motor nerve action potential, which is measured in millivolts. Therefore, the sural nerve can be difficult to monitor in obese patients or those with limb edema.

Moss and colleagues suggest that the simpler and more streamlined EPS that they describe could be used widely as a screening tool in the critically ill, thereby avoiding the need for complex and time-consuming EPS to confirm or exclude CIP/CIM. So, is it time for us to adopt this simplified approach into routine clinical practice? Introduction of a new diagnostic test requires that it be supported by evidence from prospective clinical studies, be free from harm, and be truly superior to other, currently available, options [15]. Thus, it is essential that the diagnostic accuracy of any new test should be evaluated prior to introduction into clinical practice to avoid the incorporation of invalid tests or those that yield exaggerated and/or biased results [16]. No study has yet adequately validated a simplified EPS for use in a general ICU population using rigorous methodology, particularly the independent and blinded comparison of the test under evaluation against a reference standard. There is therefore a need for diagnostic accuracy studies of simplified EPS in large, representative populations of ICU patients. The study by Moss and colleagues is one step on this road.

Acknowledgments M. Smith is part funded by the National Institute for Health Research University College London Hospitals Biomedical Research Centre.

Conflicts of interest M. Smith is Neuroscience Section Editor of Intensive Care Medicine. N. Latronico is the principal investigator of the CRIMYNE-2 study, a multicenter diagnostic accuracy study of simplified electrophysiological study. 


\section{References}

1. Latronico N (2003) Neuromuscular alterations in the critically ill patient: critical illness myopathy, critical illness neuropathy, or both? Intensive Care Med 29:1411-1413

2. Stevens RD, Dowdy DW, Michaels RK, Mendez-Tellez PA, Pronovost PJ, Needham DM (2007) Neuromuscular dysfunction acquired in critical illness: a systematic review. Intensive Care Med 33:1876-1891

3. Latronico N, Fenzi F, Recupero D, Guarneri B, Tomelleri G, Tonin P, De Maria G, Antonini L, Rizzuto N, Candiani A (1996) Critical illness myopathy and neuropathy. Lancet 347:1579-1582

4. Connolly BA, Jones GD, Curtis AA, Murphy PB, Douiri A, Hopkinson NS, Polkey MI, Moxham J, Hart N (2013) Clinical predictive value of manual muscle strength testing during critical illness: an observational cohort study. Crit Care 17:R229

5. Latronico N, Bolton CF (2011) Critical illness polyneuropathy and myopathy: a major cause of muscle weakness and paralysis. Lancet Neurol 10:931-941

6. Stevens RD, Marshall SA, Cornblath DR, Hoke A, Needham DM, de Jonghe B, Ali NA, Sharshar T (2009) A framework for diagnosing and classifying intensive care unit-acquired weakness. Crit Care Med 37(Suppl):299-308
7. Koch S, Spuler S, Deja M, Bierbrauer J, Dimroth A, Behse F, Spies CD, Wernecke KD, Weber-Carstens S (2011) Critical illness myopathy is frequent: accompanying neuropathy protracts ICU discharge. J Neurol Neurosurg Psychiatry 82:287-293

8. Guarneri B, Bertolini G, Latronico N (2008) Long-term outcome in patients with critical illness myopathy or neuropathy: the Italian multicentre CRIMYNE study. J Neurol Neurosurg Psychiatry 79:838-841

9. Moss M, Yang M, Macht M, Sottile P, Gray L, McNulty M, Quan D (2014)

Screening for critical illness polyneuromyopathy with single nerve conduction studies. Intensive Care Med. doi:10.1007/s00134-014-3251-6

10. Batt J, dos Santos CC, Cameron JI, Herridge MS (2013) Intensive care unitacquired weakness: clinical phenotypes and molecular mechanisms. Am J Respir Crit Care Med 187:238-246

11. Maffiuletti NA, Roig M, Karatzanos E, Nanas S (2013) Neuromuscular electrical stimulation for preventing skeletal-muscle weakness and wasting in critically ill patients: a systematic review. BMC Med 11:137

12. Latronico N, Shehu I, Guarneri B (2009) Use of electrophysiologic testing. Crit Care Med 37:S316-S320
13. Qaseem A, Humphrey LL, Chou R, Snow V, Shekelle P (2011) Use of intensive insulin therapy for the management of glycemic control in hospitalized patients: a clinical practice guideline from the American College of Physicians. Ann Intern Med 154:260-267

14. Latronico N, Bertolini G, Guarneri B, Botteri M, Peli E, Andreoletti S, Bera P, Luciani D, Nardella A, Vittorielli E, Simini B, Candiani A (2007) Simplified electrophysiological evaluation of peripheral nerves in critically ill patients: the Italian multi-centre CRIMYNE study. Crit Care 11:R11

15. Choosing Wisely. An initiative of the ABIM foundation. http://www. choosingwisely.org (2014). Accessed 30 Jan 2014

16. Bossuyt PM, Reitsma JB, Bruns DE, Gatsonis CA, Glasziou PP, Irwig LM, Lijmer JG, Moher D, Rennie D, de Vet HC (2003) Towards complete and accurate reporting of studies of diagnostic accuracy: the STARD initiative. BMJ 326:41-44 